\section{LA-UR-03- 3155}

Approved for public release;

distribution is unlimited.

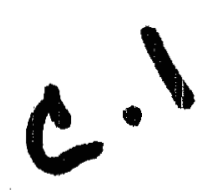

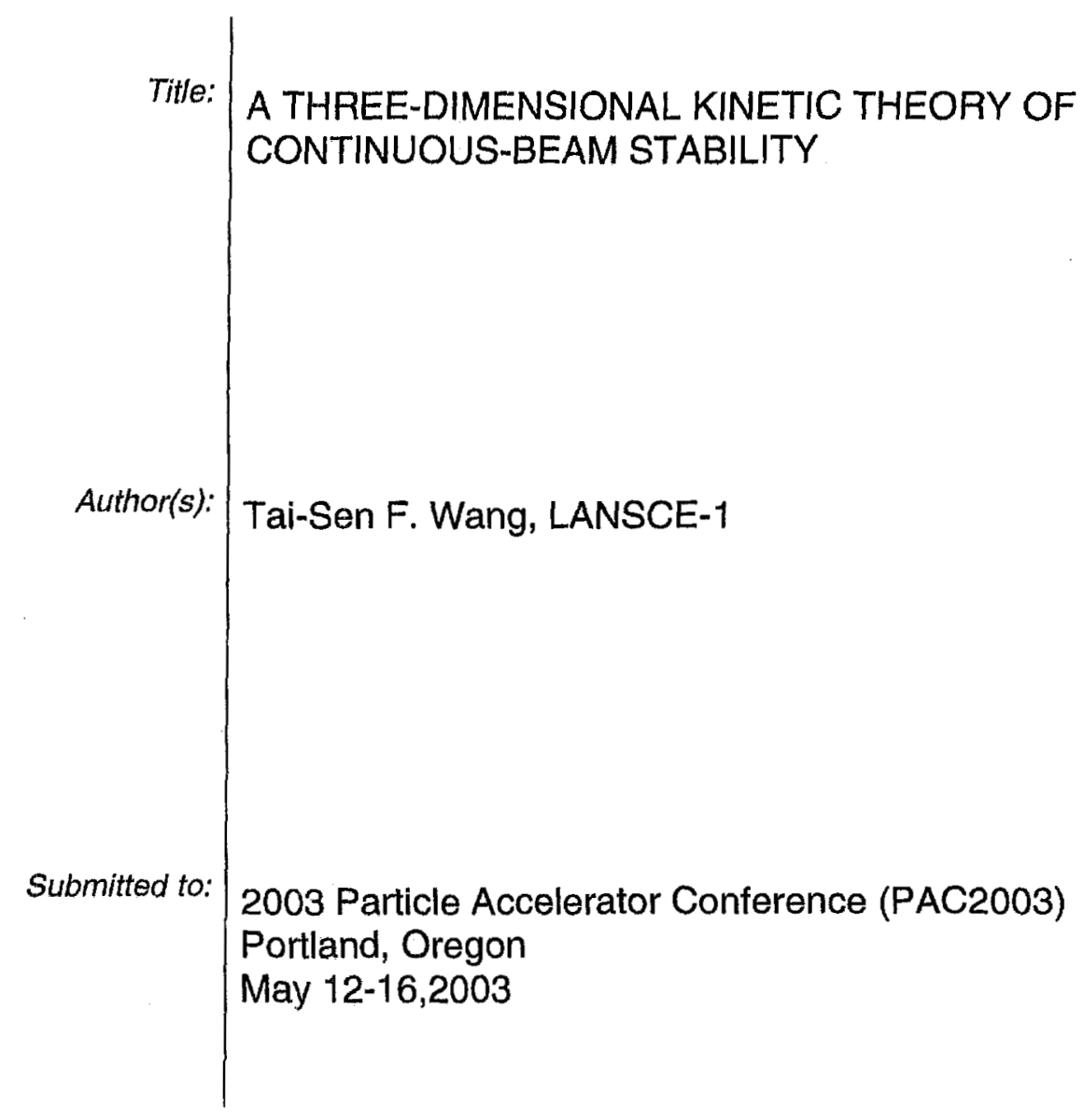

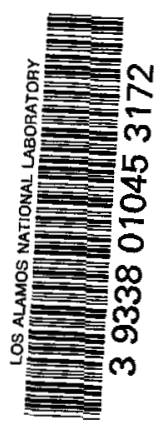

\section{NOTIONAL LABORATORY}

Los Alamos National Laboratory, an affirmative action/equal opportunity employer, is operated by the University of California for the U.S. Department of Energy under contract W-7405-ENG-36. By acceptance of this article, the publisher recognizes that the U.S. Government retains a nonexclusive, royalty-free license to publish or reproduce the published form of this contribution, or to allow others to do so, for U.S. Government purposes. Los Alamos National Laboratory requests that the publisher identify this article $\equiv$ lork performed under the auspices of the U.S. Department of Energy. Los Alamos National Laboratory strongly supports acaden $\square$ sedom and a researcher's right to publish; as an institution, however, the Laboratory does not endorse the viewpoint of a publication or guarantee its technical correctness. 


\title{
A THREE-DIMENSIONAL KINETIC THEORY OF CONTINUOUS-BEAM STABILITY *
}

\author{
Tai-Sen F. Wang ${ }^{\dagger}$, LANL, Los Alamos, NM 87545, USA
}

\begin{abstract}
This work is a three-dimensional stability study based on the modal analysis for a continuous beam of KapchinskijVladimirskij (KV) distribution. The analysis is carried out self-consistently within the context of linearized VlasovMaxwell equations and electrostatic approximation. The emphasis is on investigating the coupling between longitudinal and transverse perturbations in the high-intensity region. The interaction between the transverse modes supported by the KV distribution and those modes sustainable by the cold beam is examined. We found two classes of coupling modes that would not exist if the longitudinal and the transverse perturbations, are treated separately. The effects of wall impedance on beam stability is also studied and numerical examples are presented.
\end{abstract}

\section{INTRODUCTION}

In a customary stability analysis of a continuous beam in an accelerator or storage ring, longitudinal and transverse effects are treated separately, an approximation that is valid because space-charge forces are relatively weak and characteristic frequencies differ by orders of magnitude. For a very intense beam like the one in the proposed heavyion fusion facility, the space-charge forces are large and all frequencies are of the order of the plasma frequency, the separated treatment of longitudinal and transverse perturbations may not be applicable. Such a concern was raised more than two decades ago in the heavy ion fusion studies. Since then, some investigations have been exploited in attempt to address the issue by improving the earlier stability theories for laminar beams or nearly laminar beams. In a study of two-dimensional, axisymmetric perturbations in a beam of KV distribution, an instability caused by the coupling between the longitudinal and transverse motion was discovered in theory.[1] Later computer simulations confirmed the prediction and found this kind of instability to be a mechanism for energy exchange between the longitudinal and transverse motions in beams with high anisotropy in temperatures. [2-4] These findings and many fine papers published afterward [5-11] mark a success in exploring the three-dimensional beam stability. However, to date, the rigorous theory, though not necessarily computer simulations, is still left in the axisymmetric geometry and the threedimensional theory remains to be improved. The purpose of this work is to extend the earlier investigation of axisymmetric modes in a KV beam to a full three-dimensional stability study. It is hoped that the approach and the results of

\footnotetext{
"Research supported by Los Alamos National Laboratory under the auspices of the US Department of Energy.

†TANG@LANL.GOV
}

this work will be helpful in the exploring and understanding of beam stability in non-axisymmetric geometry.

\section{THEORETICAL MODEL}

We consider a continuous, nonrelativistic beam of circular cross section with radius $a$ and constant particle density $\rho_{0}$ propagating inside a conducting pipe of radius $b$ and arbitrary wall impedance. A cylindrical coordinate system $(r, \varphi, z)$ is chosen such that the beam is propagating in the positive $z$ direction and the $z$ axis coincides with the central axis of the beam. The equilibrium state of the beam is maintained by a constant linear external transverse focusing force which can be represented as $M \nu_{0}^{2} r$ where $M$ is the mass of a beam particle and $\nu_{o}$ is the betatron frequency in the absence of the beam's self-field. Taking the self-field of the beam into account, one finds the relation $\nu^{2}=\nu_{0}^{2}-\left(\omega_{p}^{2} / 2\right)$, between the effective betatron frequency of particles, $\nu$, and the plasma frequency, $\omega_{p}=\left(4 \pi q^{2} \rho_{0} / M\right)^{1 / 2}$, where $q$ is the charge of a beam particle. We assume the equilibrium distribution of beam particles in the phase space is described by the distribution function $f_{0}(\mathbf{x}, \mathbf{v})$ that is a product of the $\mathrm{KV}$ distribution in the transverse direction and a delta function of the longitudinal speed, i.e.

$$
f_{0}(\mathbf{x}, \mathbf{v})=\frac{\rho_{0}}{\pi} \delta\left[v_{\perp}^{2}-\nu^{2}\left(a^{2}-r^{2}\right)\right] \delta_{z}\left(v_{z}-v_{o}\right),
$$

where $v_{\perp}^{2}=v_{r}^{2}+v_{\varphi}^{2}, v_{r}, v_{\varphi}$ and $v_{z}$ are the particle's radial, azimuthal and the axial speeds, respectively, $v_{o}$ is the averaged axial speed of particles, and $\delta(x)$ is the delta function.

\section{STABILITY ANALYSIS}

The stability study here is carried out within the context of the Vlasov-Maxwell equations and the electrostatic approximation for small perturbations evolving in the linear regime. Thus, we consider small perturbations in the distribution function $f_{1}(\mathbf{x}, \mathbf{v}, t)$ and in the electric potential $\phi_{1}(\mathbf{x}, t)$ described by the linearized Vlasov-Poisson equations

$$
\frac{\partial f_{1}}{\partial t}+\mathbf{v} \cdot \frac{\partial f_{1}}{\partial \mathbf{x}}+\frac{d \mathbf{v}}{d t} \cdot \frac{\partial f_{1}}{\partial \mathbf{v}}=\frac{q}{M} \nabla \phi_{1} \cdot \frac{\partial f_{0}}{\partial \mathbf{v}},
$$

and

$$
\nabla^{2} \phi_{1}=-4 \pi q \int_{-\infty}^{\infty} \int_{-\infty}^{\infty} \int_{-\infty}^{\infty} f_{1}(\mathbf{x}, \mathbf{v}, t) d^{3} v
$$

Assuming the perturbed quantities vary in space and time according to $\left\{f_{1}, \phi_{1}\right\}=\{\tilde{f}, \tilde{\phi}\} \mathrm{e}^{i(\omega t+m \varphi-k z)}$, the linearized Vlasov-Poisson equation can be solved by integrating over the unperturbed particle orbit to yield the following differential-integral equation in the region of $r \leq a$, 


$$
\begin{aligned}
& \frac{1}{r} \frac{\partial}{\partial r}\left(r \frac{\partial \tilde{\phi}}{\partial r}\right)-\left(\frac{m^{2}}{r^{2}}+k^{2}\right) \tilde{\phi} \\
& =\frac{\omega_{p}^{2}}{a \nu^{2}} \tilde{\phi}(a) \delta(r-a)+\frac{2 \omega_{p}^{2}}{\pi}\left[i \Omega \int_{-\infty}^{\infty} \int_{-\infty}^{\infty} d v_{r} d v_{\varphi} \frac{d \delta_{\perp}}{d v_{\perp}^{2}}\right. \\
& \quad \times \int_{0}^{\infty} \tilde{\phi}\left(r^{\prime}\right)\left(\frac{1}{r^{\prime}}\right)^{m}\left(\xi_{1} \mathrm{e}^{i \theta}+\xi_{2}\right)^{m} \mathrm{e}^{-i \Omega r} d \tau \\
& \quad+\frac{k^{2}}{2} \int_{-\infty}^{\infty} \int_{-\infty}^{\infty} d v_{r} d v_{\varphi} \delta_{\perp} \int_{0}^{\infty} \tau \tilde{\phi}\left(r^{\prime}\right)\left(\frac{1}{r^{\prime}}\right)^{m} \\
& \left.\quad \times\left(\xi_{1} \mathrm{e}^{i \theta}+\xi_{2}\right)^{m} \mathrm{e}^{-i \Omega \tau} d \tau\right]
\end{aligned}
$$

where $\delta_{\perp}=\delta\left[v_{\perp}^{2}-\nu^{2}\left(a^{2}-r^{2}\right)\right], \xi_{1}=\left(v_{\perp} / \nu\right) \sin (\nu \tau)$, $\xi_{2}=r \cos (\nu \tau), \Omega=\omega-k v_{o}$ is the Doppler-shifted frequency, $r^{\prime}=\left(\xi_{1}^{2}+\xi_{2}^{2}+2 v_{r} \xi_{1} \xi_{2} / v_{\perp}\right)^{1 / 2}$, and $m=0$, $1,2, \ldots$ denotes the azimuthal harmonic number. The right hand side of Eq. (4) is zero in the region of $a<r \leq b$.

Expanding the perturbed electric potential in Eq. (4) as a sum of Jacobi polynomials $P_{l}^{(m, 0)}(x)$ according to

$$
\tilde{\phi}(r)=\left(\frac{r}{a}\right)^{m} \sum_{l=0}^{\infty} G_{l} P_{l}^{(m, 0)}\left(1-\frac{2 r^{2}}{a^{2}}\right),
$$

we can derive a recursion relation

$$
W_{l} A_{l+1}+\left(W_{l}+W_{l-1}+U_{l}\right) A_{l}+W_{l-1} A_{l-1}=0,
$$

for $l=1,2,3, \cdots$, where $G_{j}$ is indenpednt of $r, A_{l}=$ $\sum_{j=l}^{\infty}(-1)^{j+l} G_{j}$,

$$
\begin{gathered}
U_{l}=2(m+2 l)+\left(\frac{\omega_{p}}{\nu}\right)^{2}\left[B_{l-1}(m, \alpha)-B_{l}(m, \alpha)\right], \\
W_{l}=\frac{a^{2} k^{2}}{2(m+2 l+1)}\left[1+\left(\frac{\omega_{p}}{\nu}\right)^{2} \frac{\partial B_{l}(m, \alpha)}{\partial \alpha}\right], \\
B_{l}(m, \alpha)=i \int_{0}^{\infty} e^{-i \alpha x} \cos ^{m} x P_{l}^{(0, m)}(\cos 2 x) d x,
\end{gathered}
$$

and $\alpha=\Omega / \nu$. Applying the proper boundary conditions at $r=a$ together with Eq. (6) leads to the dispersion relation

$$
\left.\frac{a}{\tilde{\phi}_{o}} \frac{d \tilde{\phi}_{o}}{d r}\right|_{r=a}=m+\left(\frac{\omega_{p}}{\nu}\right)^{2}\left[1-B_{0}(m, \alpha)\right]+W_{0}+\frac{W_{0} A_{1}}{A_{0}},
$$

where the ratio $A_{1} / A_{0}$ can be expressed in terms of infinite determinants or a continuous fractions, and

$$
\begin{aligned}
\tilde{\phi}_{o}(r) \sim & I_{m}(k r) K_{m}(k b)-I_{m}(k b) K_{m}(k r) \\
& -i \mathcal{Z}\left[I_{m}(k r) K_{m}^{\prime}(k b)-K_{m}(k r) I_{m}^{\prime}(k b)\right],
\end{aligned}
$$

is the potential external to the beam derive from solving Eq. (4) in the region of $a<r \leq b$. Here, $I_{n}(x)$ and $K_{n}(x)$ are the $n$th order modified Bessel functions of the first and the second kinds, respectively, the prime indicates the derivative with respect to the argument, $\mathcal{Z}=\omega Z /(c k)$, $Z$ is the wall impedance, and $c$ is the speed of light,

For $k=0$, the recursion relation (6) reduces to the dispersion relation $U_{j}=0$ for the transverse modes discussed by Gluckstern before.[12] When $m=0$, the Jacobi polynomial becomes Legendre polynomials and the dispersion relation (10) reduces to the dispersion relation for axisymmetrical modes studied previously in Ref. 1. Taking the limit of $\nu \rightarrow 0$ in Eq. (10), one finds the cold-beam dispersion relation derived long time ago.[13] The customary dispersion relation of the "usual dipole mode"[14] in a continuous nonrelativistic beam without axial momentum spread can be obtained from Eq. (10) by considering the limit of $k b \ll 1$ for $m=1$.

The roots of the dispersion relation (10) fall into three classes: (i) the ones that approach the pure transverse modes, i.e., the solutions of $U_{j}=0$, when $k \rightarrow 0$, (ii) the "high-frequency coupling modes" having the limit of $\Omega \rightarrow n \nu$ when $\omega_{p} \rightarrow 0$, and (iii) the "low-frequency coupling modes" with $\Omega \rightarrow 0$ when $\omega_{p} \rightarrow 0$. Both types of "coupling modes" are fully three-dimensional perturbations and therefor vanish when $k=0$ or $m=0$ or when the longitudinal and the transverse perturbations are treated separately. The high-frequency coupling modes do not exist in the axisymmetric perturbations, and the lowfrequency coupling modes exist only in the perturbations of even and zero $m$. For the low-frequency coupling modes, $\Omega^{2}$ is roughly proportional to the azimuthal average of the perturbed field. The "usual transverse mode" found in the customary analyses[14] are similar to the lowest radial modes in class (i). When there is no strong necessity to distinguish the roots among the solutions of $U_{j}=0$, we shall use the notation $T_{m, j}$ to represent the whole family of solutions associated with $U_{j}=0$ for the $m$ th azimuthal harmonic. The usual transverse modes will be referred to as the $T_{m, 0}$ modes, the high-frequency coupling modes will be designated as $C_{m, j}$ modes, and the low-frequency coupling modes will be referred to as $L_{m, n}$ modes for $n \geq 1$, in the order of their first appearance in solving the dispersion relation using the $(2 n-1) \times(2 n-1)$ determinants.

\section{NUMERICAL EXAMPLE}

Here, we present a numerical example of the solutions to the dispersion relation (10) for some low radial modes associated with the dipole $(m=1)$ perturbation. Readers are referred to Ref. 1 for the numerical results of the axisymmetric modes. We consider only the case of $b / a=1.5$ and $k a=1$ in order to narrow down the parameter space. The infinite determinants in the dispersion relation have to be truncated to finite ranks for a practical numerical computation. We limit our study to the first 16 transverse modes, i.e up to the $T_{1,3}$ modes, obtainable in the computation using the $3 \times 3$ truncation of the infinite determinants. The roots are computed using the determinants truncated at the fourth rank for improved accuracy. We have calculated $\Omega / \nu_{o}$ from $\nu / \nu_{o}=0$ to $\nu / \nu_{o}=1$. The real part of $\Omega / \nu_{o}$ is shown in Fig. 1 as a function of tune depression $\nu / \nu_{o}$. The highintensity, low-frequency region is shown in Fig. 2, where the real part of $\Omega^{2} / \nu_{o}^{2}$ is plotted as a function of $\nu / \nu_{o}$.

As shown in Fig. 1, that for all modes, the values of 
$\Omega / \nu_{o}$ start from the solutions of $U_{j}=0(j=1,2$, and 3 here), i.e. from $1,3,5$, and 7 , at $\nu=\nu_{o}$, and decrease when the beam intensity increases. When $\nu \rightarrow 0$, the $T_{1,0}$ mode approaches the cold-beam limit, while the $\Omega / \nu_{o}$ of the up. per $T_{1,2}$ and $T_{1,1}$ modes approach 2 , and the frequencies of all other modes approach zero.

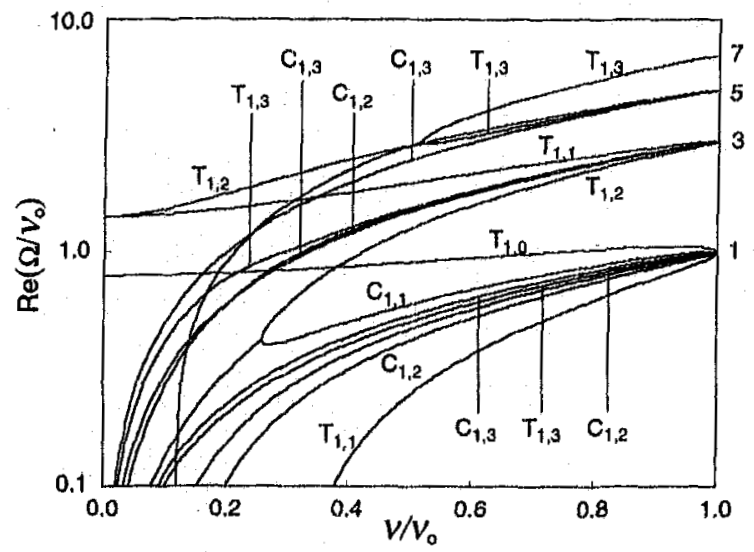

Figure 1: The real part of $\Omega / \nu_{o}$ for some $m=1$ modes as a function of $\nu / \nu_{o}$ for $k a=1.0, b / a=1.5$, and $Z=0$.

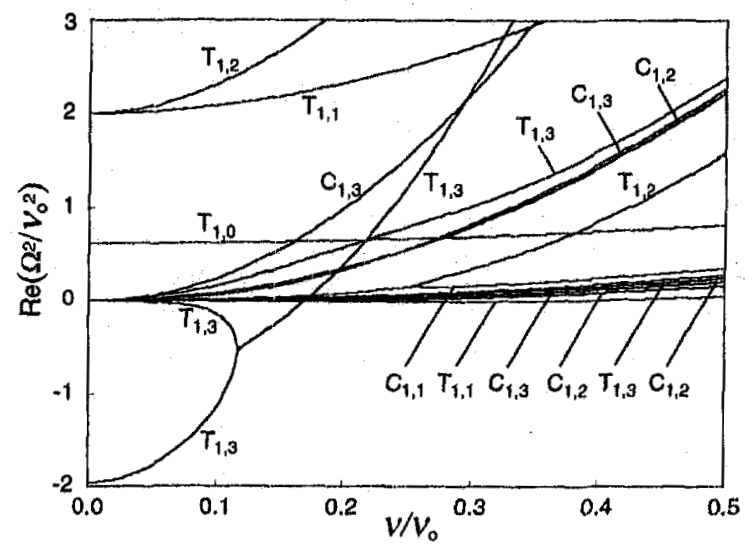

Figure 2: The real part of $\Omega^{2} / \nu_{o}^{2}$ for some $m=1$ modes as a function of $\nu / \nu_{0}$ in the low-frequency, high tune depression region for $k a=1.0, b / a=1.5$, and $Z=0$.

Mode coupling appears in the high-density, low frequency region as confluences of modes where two or more modes have the same real part of frequency. Up to the $3 \times 3$ truncation of the infinite determinants, three confluences are found: the confluence of $T_{1,0}$ and $T_{1,2}$ near $\nu=0.38 \nu_{o}$, the confluence of $C_{1,1}$ and $T_{1,2}$ between $\nu=0$ and $\nu=0.26 \nu_{o}$, and the confluence of two upper $T_{1,3}$ modes between $\nu=0.12 \nu_{o}$ and $\nu=0.52 \nu_{0}$. The frequencies in the confluent regions form complex conjugate pairs indicating possible instability. In addition, the lower $T_{1,1}$ and two lower $C_{1,2}$ modes are found to be unstable in the high-intensity region. When varying the wall impedance, we found that only the usual dipole mode, the $T_{1,0}$ mode, is appreciably influenced. The maximal growth rate occurs near $\nu \approx 0$ as expected. In the case considered here, we found that for $T_{1,0}$ mode, the maximum of $\left|\operatorname{Im}\left(\Omega / \nu_{o}\right)\right|$ has the values of $0.0,0.034,0.066,0.092$ for $\mathcal{Z}=0.0,0.1,0.2$, and 0.3 , respectively.

\section{CONCLUSIONS}

We have studied the three-dimensional stability of a continuous beam of KV distribution. The analysis is carried out self-consistently within the context of linearized Vlasov-Maxwell equations and electrostatic approximation. Mode confluence is found to occur in the highintensity region indicating possible instability. A few other modes are also found to be unstable in the high-intensity region. Since not all instability in a KV beam are realistic, computer simulations are suggested for further investigation. Two classes of coupling modes are discovered. These coupling modes would not exist if the longitudinal and the transverse perturbations are treated separately. The effects of wall impedance on beam stability is also studied. The results indicate that only the usual transverse modes are appreciably affected by the wall impedance.

\section{REFERENCES}

[1] T. F. Wang and L. Smith, Particle Accelerators, 12 (1982) 247.

[2] I. Haber, D. A. Callahan, A. Friedman, D. P. Grote and A. B. Langdon, Fusion Engn. and Design, 32-33 (1996) 169.

[3] S. M. Lund, D. A. Callahan, A. Friedman, D. P. Grote, I. Haber and T. F. Wang, Proc. of the XIX Int. Linear Accelerator Conf., 1998, p. 372.

[4] I. Haber, D. A. Callahan, A. Friedman, D. P. Grote, S. M. Lund and T. F. Wang, Nucl. Instrum. Methods in Phys. Res., A415 (1998) 405.

[5] S. M. Lund and R. C. Davidson, Phys. Plasmas, 5 (1998) 3028.

[6] I. Haber, A. Friedman, D. P. Grote, S. M. Lund and R. A. Kishek, Phys. Plasmas, 6 (1999) 2254.

[7] R. C. Davidson and S. Strasburg, Phys. Plasmas, 7 (2000) 2657.

[8] H. Qin, R. C. Davidson, W. W.-L. Lee and R. Kolesnikov, Nucl. Instrum. Methods in Phys. Res., A464 (2001) 477.

[9] I. Haber, A. Friedman, D. P. Grote, S. M. Lund, S. Bernal and R. A. Kishek, Nucl. Instrum. Methods in Phys. Res., A464 (2001) 343.

[10] R. C. Davidson, H. Qin, W. W.-L. Lee and S. Strasburg, Nucl. Instrum. Methods in Phys. Res., A464 (2001) 358.

[11] E. A. Startsev, R. C. Davidson and H. Qin, Phys. Plasmas, 9 (2002) 3138 .

[12] R. L. Gluckstern, Proc. 1970 Proton Linear Accelerator Conf., National Accelerator Laboratory, 1970, p. 811.

[13] W. C. Hahn, Gen. Elec. Rev., 42, 258 (1939), and S. Ramo, Phys. Rev., 56, 276 (1939).

[14] For example, see the discussion in SLAC Report SLAC-76, 1967, by M. J. Lee, F. E. Mills and P. L. Morton. 